OPEN ACCESS

Edited by:

Chee $\mathrm{Ng}$,

The University of Melbourne, Australia

Reviewed by:

Célia Fourrier,

University of Adelaide, Australia Alessio Squassina Università degli Studi di Cagliari, Italy Leszek Rudzki,

Medical University of Bialystok, Poland

*Correspondence:

Hiroshi Kunugi

hkunugi@ncnp.go.jp

Specialty section:

This article was submitted to

Mood and Anxiety Disorders,

a section of the journal

Frontiers in Psychiatry

Received: 05 August 2018 Accepted: 10 December 2018

Published: 18 January 2019

Citation:

Aizawa E, Tsuji H, Asahara T,

Takahashi T, Teraishi T, Yoshida S,

Koga N, Hattori K, Ota M and

Kunugi $H$ (2019) Bifidobacterium and

Lactobacillus Counts in the Gut

Microbiota of Patients With Bipolar

Disorder and Healthy Controls.

Front. Psychiatry 9:730

doi: 10.3389/fpsyt.2018.00730

\section{Bifidobacterium and Lactobacillus Counts in the Gut Microbiota of Patients With Bipolar Disorder and Healthy Controls}

Emiko Aizawa ${ }^{1,2}$, Hirokazu Tsuji ${ }^{3}$, Takashi Asahara ${ }^{3}$, Takuya Takahashi $^{3}$, Toshiya Teraishi ${ }^{1}$, Sumiko Yoshida ${ }^{4}$, Norie Koga ${ }^{1}$, Kotaro Hattori ${ }^{1}$, Miho Ota ${ }^{1}$ and Hiroshi Kunugi ${ }^{1 *}$

${ }^{1}$ Department of Mental Disorder Research, National Institute of Neuroscience, National Center of Neurology and Psychiatry, Tokyo, Japan, ${ }^{2}$ Department of Human Life Science, Nagoya University of Economics, Aichi, Japan, ${ }^{3}$ Yakult Central Institute, Tokyo, Japan, ${ }^{4}$ Department of Psychiatry, National Center of Neurology and Psychiatry Hospital, Tokyo, Japan

Background: Although the pathophysiology of bipolar disorder remains elusive, growing evidence suggests the beneficial effects of Bifidobacterium and Lactobacillus in the gut microbiota on stress response and depressive symptoms. In the present study, we examined Bifidobacterium and Lactobacillus counts for association with bipolar disorder and serum cortisol levels.

Methods: Bacterial counts in fecal samples were examined in 39 patients with bipolar disorder according to the Diagnostic and Statistical Manual of Mental Disorders, 4th edn. and 58 healthy controls using bacterial rRNA-targeted reverse transcription-quantitative polymerase chain reaction.

Results: No significant difference was found in either bacterial counts between the two groups. However, we found a significantly negative correlation between Lactobacillus counts and sleep $(\rho=-0.45, P=0.01)$. Furthermore, a significant negative correlation was found between Bifidobacterium counts and cortisol levels $(\rho=-0.39, P=0.02)$ in the patients, although such a correlation was not found for Lactobacillus counts.

Conclusions: Our results suggest that Bifidobacterium or Lactobacillus counts may not play a major role in the pathophysiology of bipolar disorder in our sample. However, the observed negative correlation between Lactobacillus counts and sleep and that between Bifidobacterium counts and serum cortisol levels point to the possible roles of these bacteria in sleep and stress response of the patients.

Keywords: Bifidobacterium, Lactobacillus, bipolar disorder, cortisol levels, stress response

\section{INTRODUCTION}

Increasing evidence suggests that alterations in the gut microbiota are involved in stress-related psychiatric disorders (1-4). Animal studies have revealed that the enteric microbiota affects neural activity, neuronal pathways, emotional state, immune system/inflammation, stress responses (i.e., hypothalamic-pituitary-adrenal [HPA] axis activity), and cognitive function in the host. A recent 
study reported that fecal microbiota transplantation from depressed patients causes depression-like behaviors in mice (511).

Bipolar disorder is a serious mental disorder that markedly affects an individual's daily life. The efficacy of current pharmacological treatment is inadequate; in brief, residual mood symptoms often remain after initial treatment and subsequent recurrence is frequent, which might be due to the lack of knowledge regarding the neurobiological mechanisms underlying the disorder $(12,13)$. Nonetheless, bipolar disorder is considered to be caused by abnormal monoamine functions, dysregulation of the hypothalamus-pituitary-adrenal (HPA) axis, and chronic inflammation (14-17), all of which may be mediated by alterations in the gut microbiota $(8,9,11,18)$.

Amongst the many bacterial genera in the gut, Bifidobacterium and Lactobacillus are considered to be probiotics, which exert beneficial effects on physical and mental health such as psychiatric symptoms. For example, Messaoudi et al. (19) reported that healthy volunteers, who consumed a combination of Bifidobacterium longum R0175 and Lactobacillus helveticus R0052, exhibited less distress symptoms and showed a decrease in urinary free cortisol levels (19), suggesting ameliorating effects of the probiotics on the stress-related hormonal system. The same probiotics also had a beneficial effect on brain plasticity in mice exposed to chronic stress (7). Desbonnet et al. (20) reported that Bifidobacterium infantis treatment normalized the immune response and ameliorated depression-like behavior in a rat maternal separation model of depression. Although direct evidence in psychiatric patients is still scarce, we have recently reported that Bifidobacterium and Lactobacillus counts tend to be lower in patients with major depressive disorder than in healthy controls, supporting the possible role of these bacteria in the pathophysiology of mood disorders (21). Furthermore, a randomized clinical trial showed that taking probiotic capsules (L. acidophilus, L. casei, and Bifidobacterium bifidum) had a beneficial effect on depressive symptoms, glucose metabolism, and antioxidant action (1).

Recently, several research groups have reported gut microbiota in patients with bipolar disorder (22). Evans et al. (23) examined 115 patients with bipolar disorder and 64 controls, recruited from the University of Michigan, using $16 \mathrm{~S}$ ribosomal RNA (rRNA) gene sequence analysis and Operational Taxonomical Unit (OTU) level analysis. They found significantly decreased fractional representation of Faecalibacterium in bipolar disorder patients (23). The same research group compared the gut microbiota between 46 atypical antipsychotictreated and 69 untreated patients and found differences in OTU levels (24). Painold et al. (25) performed 16S rRNA gene sequencing of stool samples from 32 bipolar individuals and 10 controls recruited from the Medical University of Graz, Austria. They reported that the phylum Actinobacteria and the class Coriobacteria were significantly more abundant in bipolar patients than in controls, while Ruminococcaceae and Faecalibacterium were more abundant in healthy controls. That

Abbreviations: HPA, hypothalamic-pituitary-adrenal; NCNP, National Center of Neurology and Psychiatry. the results of the previous two study groups were inconsistent, warrants further investigation. Additionally, given that similar studies have not been conducted with Asian bipolar patients, warrants further research in Asian populations, particularly because dietary differences have a substantial impact on gut microbiota (25). In the current study, we examined whether Bifidobacterium and Lactobacillus counts are altered in Japanese patients with bipolar disorder in comparison with healthy controls. We focused on these two bacteria because research has suggested that they have a beneficial effect on pathological factors related to mood disorders $(1,7,19,20,26)$. In addition, we examined the possible correlation between the bacterial counts and clinical variables such as severity of symptoms, medication, and serum cortisol levels.

\section{MATERIALS AND METHODS \\ Participants}

We recruited 39 patients with bipolar disorder (13 bipolar I and 26 bipolar II) and 58 healthy controls from the outpatient clinic at the National Center of Neurology and Psychiatry (NCNP) hospital and from the local community (Western Tokyo) through the NCNP website and local magazine advertisements. The majority of the controls had participated in our previous study (21). All participants were biologically unrelated Japanese individuals who were screened for eligibility by a research psychiatrist using the Japanese version of the Mini-International Neuropsychiatric Interview (27, 28), and an additional unstructured interview, to obtain information on education, illness history, psychiatric medication, height and weight, comorbid medical conditions, and recent use of antibiotics. The diagnosis of bipolar disorder was made by a board-certificated research psychiatrist, according to the Diagnostic and Statistical Manual of Mental Disorders, 4th ed. (DSM-IV) (29), based on information obtained from the interviews and medical records, if available. Depressive symptoms of patients with bipolar disorder were rated using the 17-item version Hamilton Depression Rating Scale (HAMD) (30), while the Young Mania Rating Scale was used to assess manic symptoms (31). The control group was screened to exclude candidates with a current or past history of psychiatric illness or contact with psychiatric services. Exclusion criteria for both patients and control groups included a prior medical history of central nervous system disease, including epilepsy and severe head injury, substance abuse or dependence, mental retardation, recent use of antibiotics, history of gastrointestinal surgery, severe congenital abnormalities, or any severe medical conditions. Among the patients with bipolar disorder $(n=36), 33$ patients were receiving pharmacological treatment, while the remaining three were not. The doses of antidepressants and antipsychotics were converted to imipramine and chlorpromazine equivalents, respectively, using the published guideline (32). Moreover, we obtained information on the patients' use of mood stabilizing medicine, such as lithium, valproate, lamotrigine, and carbamazepine. We obtained information on intake of probiotic drugs (prescribed or obtained over the counter); 9 patients were on probiotic 
medication. The present study was approved by the ethics committee of the National Center of Neurology and Psychiatry. Written informed consent was obtained from all participants prior to their enrollment in the study.

\section{Collection of Fecal Samples}

Participants were asked to place a fecal sample of approximately $1 \mathrm{~g}$ into a sample tube containing $2 \mathrm{~mL}$ of RNA stabilization solution, Ambion RNAlater ${ }^{\circledR}$ (Thermo Fisher Scientific, Waltham, MA, United States). The collected samples were kept at $4^{\circ} \mathrm{C}$ in a refrigerator. All fecal samples were subsequently sent to the Yakult Central Institute and stored at $4{ }^{\circ} \mathrm{C}$ until assay. Samples were weighed, and RNAlater ${ }^{\circledR}$ was added to the tube in order to make a 50 -fold diluted (v/w) fecal homogenate by vortex. This fecal homogenate $(200 \mu \mathrm{l})$ was then transferred into a 2 - $\mathrm{ml} \mathrm{screw-cap} \mathrm{microtube} \mathrm{and} \mathrm{centrifuged} \mathrm{at} 12,000 \times \mathrm{g}$ for $5 \mathrm{~min}$ at $4^{\circ} \mathrm{C}$, and the supernatant was discarded by decantation. The pellet was then stored at $-80^{\circ} \mathrm{C}$ until extraction of RNA.

\section{Determination of Bacterial Counts}

Bacterial counts in fecal samples were measured by investigators blinded to the clinical information. The measurements were performed using the Yakult Intestinal Flora-SCAN, which is based on a $16 \mathrm{~S}$ or $23 \mathrm{~S}$ rRNA-targeted reverse transcriptionquantitative polymerase chain reaction (RT-qPCR), to determine the composition of major gut bacterial groups according to a previously described method $(33,34)$. Briefly, total RNA fractions were extracted from the fecal samples, and three serial dilutions of the extracted RNA sample were used for bacterial rRNAtargeted RT-qPCR. The threshold cycle values in the linear range of the assay were applied to the standard curve to obtain the corresponding bacterial count in each nucleic acid sample. Those data were subsequently used to determine the number of bacteria per sample. The specificity of the RT-qPCR assay, using group-, genus-, or species-specific primers, was determined as previously described $(33,34)$. Bifidobacterium counts were determined using a single primer set. Lactobacillus counts were determined by adding organisms of six Lactobacillus subgroups (L. casei, L. gasseri, L. plantarum, L. reuteri, L. ruminis, and L. sakei) and two Lactobacillus species (L. brevis and L. fermentum) together, which were determined using separate RT-qPCR primers. Briefly, for identification of the target bacterial population in the fecal samples, $1 / 20,000,1 / 200,000$, or $1 / 2,000,000$ samples of the extracted RNA from $4 \mathrm{mg}$ of wet feces was subjected to RTqPCR. The quantification cycle values in the linear range of the assay were applied to the analytical curve, generated in the same experiment, to obtain the corresponding bacterial count using the $4^{\prime}, 6$-diamidino-2-phenylindole (DAPI) staining method in each nucleic acid sample. Bacterial counts were then converted to count per sample.

\section{Blood Sample Collection}

Fasting venous blood samples were collected between 9:00 and 10:00 a.m. from each participant. Samples were collected in a 9-ml serum separating tube and centrifuged at 3,000 rpm for $10 \mathrm{~min}$. Cortisol levels were measured via radioimmunoassay at SRL Inc. (Tokyo, Japan).

\section{Statistical Analyses}

Data are presented as means \pm standard deviation $(S D)$ unless otherwise specified. Demographic and clinical variables were compared using the chi-square test for categorical variables and the $t$-test for continuous variables. Univariate analysis of covariance (ANCOVA) was used to compare Bifidobacterium or Lactobacillus counts between patients with bipolar disorder and controls (adjusted for age and sex). Additional analyses controlling for BMI were also performed. Moreover, we performed ANCOVA, controlling for age, sex, and diagnosis in order to examine the influence of bacterial counts on levels of cortisol. Partial correlation analysis (adjusted for age and sex) was used to examine the partial correlations between bacterial counts and other variables such as HAM-D scores and cortisol levels. Cramer's $V$ was calculated as a measure of effect size for $\chi^{2}$ tests, $d$ for $t$-tests, $\eta^{2}$ for ANOVA, and partial $\eta^{2}$ for ANCOVA. Differences were considered statistically significant when the two-tailed $P$ value was $<0.05$. Analysis was performed using the Statistical Package for the Social Sciences version 21.0 (IBM Corp, Chicago, Illinois, USA).

\section{RESULTS}

\section{Characteristics of Study Participants}

Table 1 lists the demographic and clinical characteristics of the participants. No significant differences were observed in age, sex, body mass index (BMI), or years of education between the patient and control groups. The number of patients with depressed, manic, euthymic, or mixed episode according to the standard cut-off scores (HAM-D17 score of 7 and YMRS score of 7) were $23,2,13$, and 1 , respectively. Thus, the majority of the subjects were depressed or euthymic and there were only three patients whose YMRS score was eight or more.

\section{Comparison of Bacterial Counts}

Figure 1 depicts comparisons of Bifidobacterium and Lactobacillus counts between the patients and controls. No significant differences were found in Bifidobacterium $(d f=1,92$; $F=0.34, P=0.56$, Partial $\eta^{2}=0.004$ ) or Lactobacillus counts $\left(d f=1,92 ; F=0.14, P=0.71\right.$, Partial $\left.\eta^{2}=0.002\right)$ between the two groups. Even when male and female were examined separately, there was no significant difference in bacterial counts between the patients and controls (Supplementary Figure 1). When bipolar I and II patients were separately compared with controls, there was no significant difference in Bifidobacterium counts for bipolar I $\left(d f=1,66 ; F=0.05, P=0.83\right.$, Partial $\eta^{2}$ $=0.001)$ or for bipolar II $(d f=1,79 ; F=0.83, P=0.36$, Partial $\left.\eta^{2}=0.01\right)$ or in Lactobacillus counts for bipolar I $(d f=1,66 ; \mathrm{F}$ $=0.46, P=0.50$, Partial $\left.\eta^{2}=0.01\right)$ or for bipolar II $(d f=1,79 ; F$ $=0.86, P=0.36$, Partial $\eta^{2}=0.01$ ). When BMI was additionally controlled for, the results were essentially unchanged (data not shown).

\section{Bacterial Counts and Symptom Scores}

In the patient group, there was no significant partial correlation (adjusted for age and sex) between bacterial counts and HAMD 17 total score (for Bifidobacterium: $\rho=-0.06, P=0.72$; 
TABLE 1 | Demographic and clinical characteristics of the participants.

\begin{tabular}{|c|c|c|c|}
\hline & $\begin{array}{l}\text { Bipolar } \\
\text { disorder } \\
(n=39)\end{array}$ & $\begin{array}{l}\text { Control } \\
(n=58)\end{array}$ & $\begin{array}{l}\text { Bipolar disorder } \\
\text { vs. control }\end{array}$ \\
\hline Male (\%) & $17(44)$ & $22(38)$ & $\begin{array}{l}\chi^{2}=0.31, d f=1 \\
P=0.58, \text { Cramer's } \\
V=0.06\end{array}$ \\
\hline Age, years: & $40.3 \pm 9.2$ & $43.1 \pm 12.9$ & $\begin{array}{l}\mathrm{t}=1.23, d f=94.6 \\
P=0.22, d=0.24\end{array}$ \\
\hline Education, years & $15.3 \pm 3.3$ & $15.2 \pm 2.7$ & $\begin{array}{l}\mathrm{t}=-0.14, d f=95 \\
P=0.89, d=0.03\end{array}$ \\
\hline $\begin{array}{l}\text { Body mass index } \\
\text { (BMI) }\end{array}$ & $23.9 \pm 4.7$ & $22.4 \pm 3.8$ & $\begin{array}{l}\mathrm{t}=-1.71, \mathrm{df}= \\
69.9, P=0.09 \\
d=0.36\end{array}$ \\
\hline $\begin{array}{l}\text { HAM-D } 17 \text { total } \\
\text { score }\end{array}$ & $10.3 \pm 7.0$ & N.A. & \\
\hline $\begin{array}{l}\text { Young Mania } \\
\text { Rating Scale }\end{array}$ & $2.1 \pm 3.5$ & N.A. & \\
\hline $\begin{array}{l}\text { Age at onset, } \\
\text { years }\end{array}$ & $28.2 \pm 9.4$ & N.A. & \\
\hline \multicolumn{4}{|l|}{$\begin{array}{l}\text { Medication n (\%) } \\
\text { mg/day }\end{array}$} \\
\hline $\begin{array}{l}\text { Antipsychotic } n= \\
13(33 \%)\end{array}$ & $182.9 \pm 179.9$ & N.A & \\
\hline $\begin{array}{l}\text { Antidepressant }{ }^{\# \#} n \\
=12(31 \%)\end{array}$ & $204.3 \pm 125.8$ & N.A & \\
\hline $\begin{array}{l}\text { Lithium } n=16 \\
(41 \%)\end{array}$ & $418.8 \pm 240.1$ & N.A & \\
\hline $\begin{array}{l}\text { Sodium Valproate } \\
n=8(21 \%)\end{array}$ & $725.0 \pm 399.1$ & N.A & \\
\hline $\begin{array}{l}\text { Lamotrigine } n= \\
13(33 \%)\end{array}$ & $186.5 \pm 123.2$ & N.A & \\
\hline $\begin{array}{l}\text { Carbamazepine } n \\
=4(10 \%)\end{array}$ & $325.0 \pm 221.7$ & N.A & \\
\hline
\end{tabular}

Values are mean \pm standard deviation.

N.A, not applicable; HAM-D, Hamilton Depression Rating Scale.

\#: Chlorpromazine equivalent dose in patients with antipsychotic medication (Bipolar disorder, $n=13)$.

\#\#: Imipramine equivalent dose in patients with antidepressant medication (Bipolar disorder, $n=12$ ).

Drug free, $n=3$.

for Lactobacillus: $\rho=-0.24, P=0.16)$ or between bacterial counts and YMRS total score (for Bifidobacterium: $\rho=0.11, P=$ 0.53; for Lactobacillus: $\rho=0.25, P=0.14$ ). However, subscales of the depressive symptoms according to Seretti et al. (35) (i.e., core, sleep, activity, psychic anxiety, and somatic anxiety) were examined separately (Supplementary Table 1); we found a significantly negative correlation between Lactobacillus counts and sleep ( $\rho=-0.45, P=0.01)$ (Figure 2) (35).

\section{Bacterial Counts and Cortisol Levels}

There was no significant difference in serum cortisol levels between the patients and controls $(11.8 \pm 3.9 \mu \mathrm{g} / \mathrm{dl}$ for the patients vs. $12.7 \pm 5.3 \mu \mathrm{g} / \mathrm{dl}$ for the controls; $d f=1,90 ; F=$ $1.75, P=0.19)$. However, a significant ANCOVA main effect of cortisol levels was observed for Bifidobacterium counts $(d f=1$, $90 ; F=9.4, P=0.003$ ). Figure 3 illustrates the partial correlation between Bifidobacterium counts and cortisol levels. A significant negative correlation (age- and sex-adjusted partial correlation) was observed between Bifidobacterium counts and cortisol levels in the patients $(\rho=-0.39, P=0.02)$. A similar trend was observed in the controls, although the correlation failed to reach statistical significance $(\rho=-0.25, P=0.07)$. Lactobacillus counts exhibited no significant correlation with cortisol levels in the patients $(\rho=-0.06, P=0.56)$ or in controls $(\rho=0.14, P=$ $0.29)$.

\section{Bacterial Counts and Medication}

There was no significant partial correlation (age- and sexadjusted) of bacterial counts with dose of antipsychotics (Bifidobacterium: $\rho=-0.09, P=0.79$; Lactobacillus: $\rho=0.17, P$ $=0.62$ ) or antidepressants (Bifidobacterium: $\rho=0.01, P=0.98$; Lactobacillus: $\rho=0.07, P=0.86)$. No significant differences were observed in Bifidobacterium ( $d f=1,34 ; F=0.57, P=0.46$ ) or Lactobacillus counts ( $d f=1,34 ; F=1.08, P=0.31)$ between users and non-users of any mood stabilizer.

As described above, we included 9 patients who received probiotic agents, which may have minimized the difference in bacterial counts between patients and controls. Even after excluding these 9 patients, however, there was no significant difference for either bacterial count (for Bifidobacterium: $d f=$ 1, 83; $F=0.002, P=0.96$, Partial $\eta^{2}=0.00$; for Lactobacillus: $d f$ $=1,83 ; F=0.05, P=0.82$, Partial $\left.\eta^{2}=0.001\right)$.

\section{DISCUSSION}

The aim of this study was to first determine whether Bifidobacterium and Lactobacillus counts in the gut microbiota differ between patients with bipolar disorder and healthy controls. We then examined the correlations between these Bifidobacterium and Lactobacillus counts and depressive symptoms and serum cortisol levels. We observed no significant difference in Bifidobacterium or Lactobacillus counts between the two groups. These counts showed no significant correlation with overall severity of manic or depressive symptoms. However, there was a negative correlation between Lactobacillus counts and sleep. We found a negative correlation between Bifidobacterium counts and cortisol levels in the patients.

To our knowledge, this is the first study that focused on Bifidobacterium and Lactobacillus counts in the gut microbiota of bipolar patients using the RT-qPCR method. Notably, unlike the current RT-qPCR method, the ordinary sequencing-based (OTU) level analysis cannot precisely obtain data on Lactobacillus counts. Since we previously found reduced counts for both bacteria in patients with major depressive disorder compared with controls (21), we expected similar findings in bipolar patients. However, we found no significant difference in bacterial counts between bipolar patients and controls, suggesting that Bifidobacterium and Lactobacillus counts in the gut may not play a major role in the pathophysiology of bipolar disorder in our sample. Our results are consistent with Evans et al. (23) who reported no significant difference in the ratio of Bifidobacterium counts between bipolar patients and controls based on their OTU analysis. Painold et al. (25) reported a significant difference in the phylum Actinobacteria to which Bifidobacterium belongs; however, they did not report Bifidobacterium counts $(23,25)$. A 

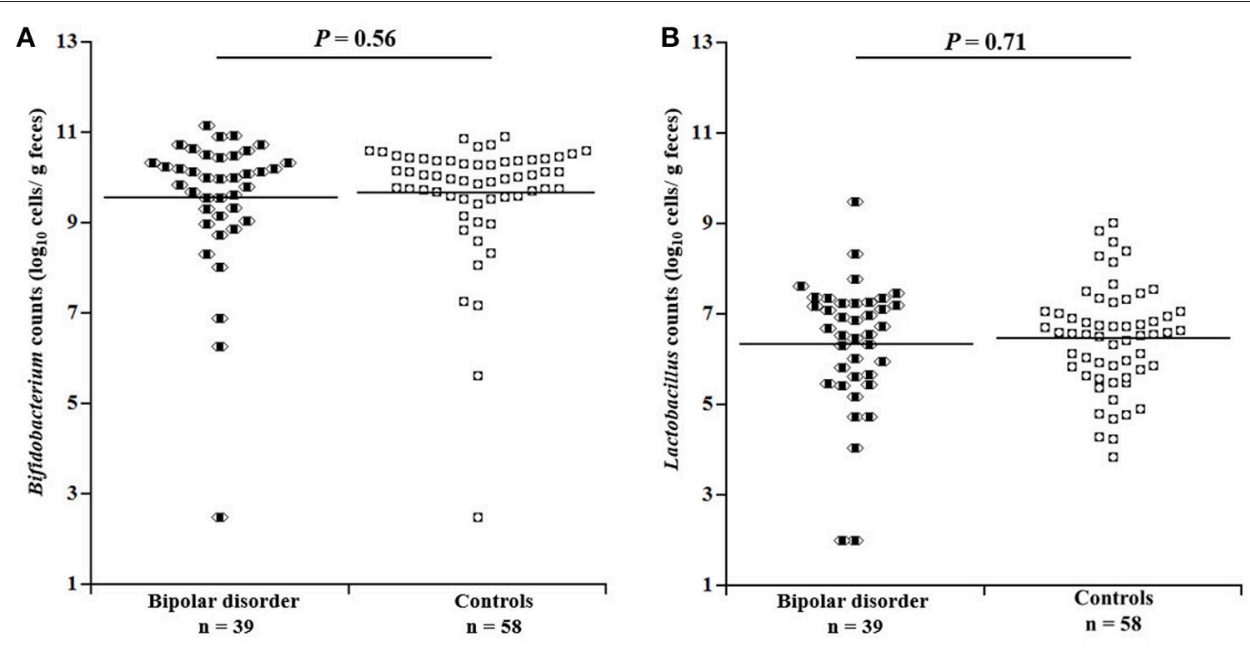

FIGURE 1 | Comparison of bacterial counts in the gut microbiota between patients with bipolar disorder and controls. No significant differences were observed between patients and controls with respect to either $(\mathbf{A})$ Bifidobacterium ( $d f=1,92 ; F=0.34, P=0.56$, Partial $\rho^{2}=0.004$, ANCOVA) or (B) Lactobacillus $(d f=1$, 92; $F=0.14, P=0.71$, Partial $\rho^{2}=0.002$ ) counts.

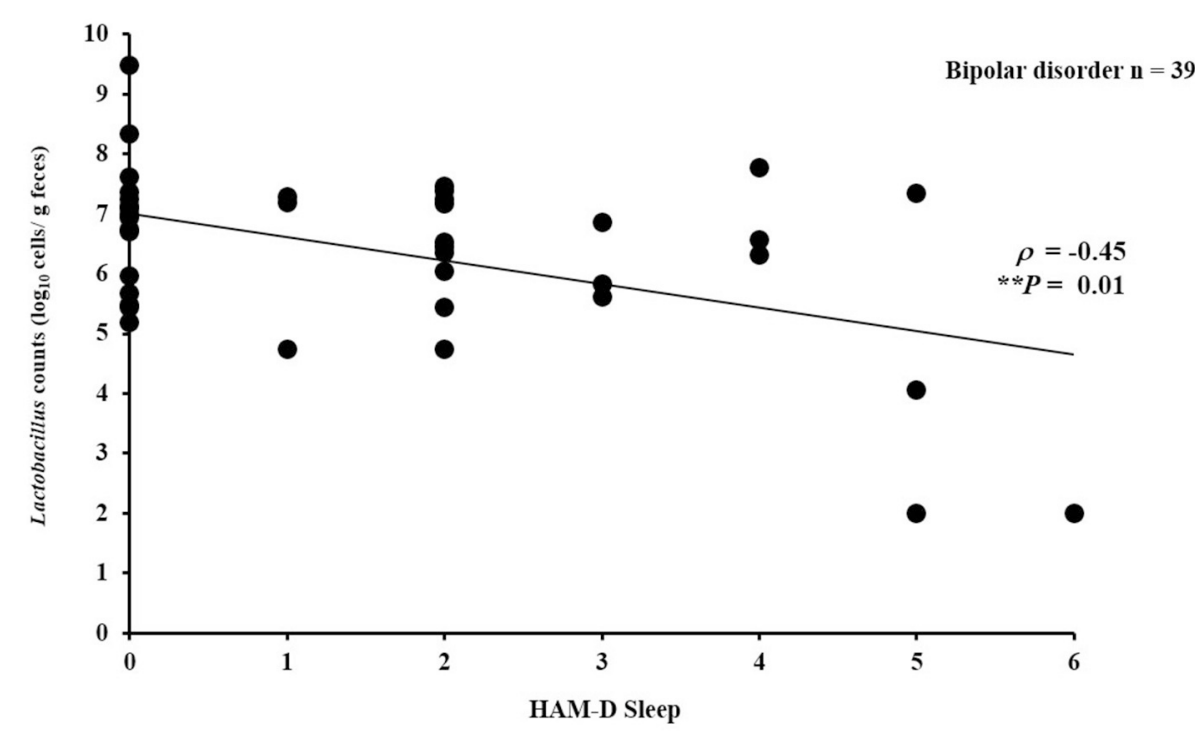

FIGURE 2 | Correlation between Lactobacillus counts and sleep (HAM-D subscale). Lactobacillus counts exhibited a significantly negative correlation with sleep (HAM-D subscale) in the patients with bipolar disorder $(\rho=-0.45, P=0.01)$.

limitation in our sample is that most patients were being treated with medication and their disease severity was relatively mild (mean HAMD-17 score of $10.3 \pm 7.0$ and Young mania rating scale score of $2.1 \pm 3.5$ ), which may have contributed to the negative results.

We found no significant correlation between overall HAMD17 or YMRS score and bacterial counts. However, there was a negative correlation between the Lactobacillus counts and sleep on the HAM-D scale. In concordance with this, Takada et al. reported that Lactobacillus casei had beneficial effects on stressinduced sleep disturbance in humans (36). Miyazaki et al. also found that Lactobacillus brevis had beneficial effects on sleep rhythms in mice (37). Together with our finding, increasing
Lactobacillus counts in the gut may be beneficial to sleep disturbances in bipolar disorder.

Although a recent study reported an association between atypical antipsychotics and specific representation of gut bacterial families in the microbiota, such as Lachnospiraceae, Akkermansia, and Sutterella in bipolar patients (24), we found no evidence for the effects of any psychotropic medications on Bifidobacterium or Lactobacillus counts in our subjects.

Interestingly, in the patients, Bifidobacterium counts correlated negatively with serum cortisol levels, a hormone critically involved in the HPA axis and commonly regarded as a biological indicator of stress in most psychobiological 

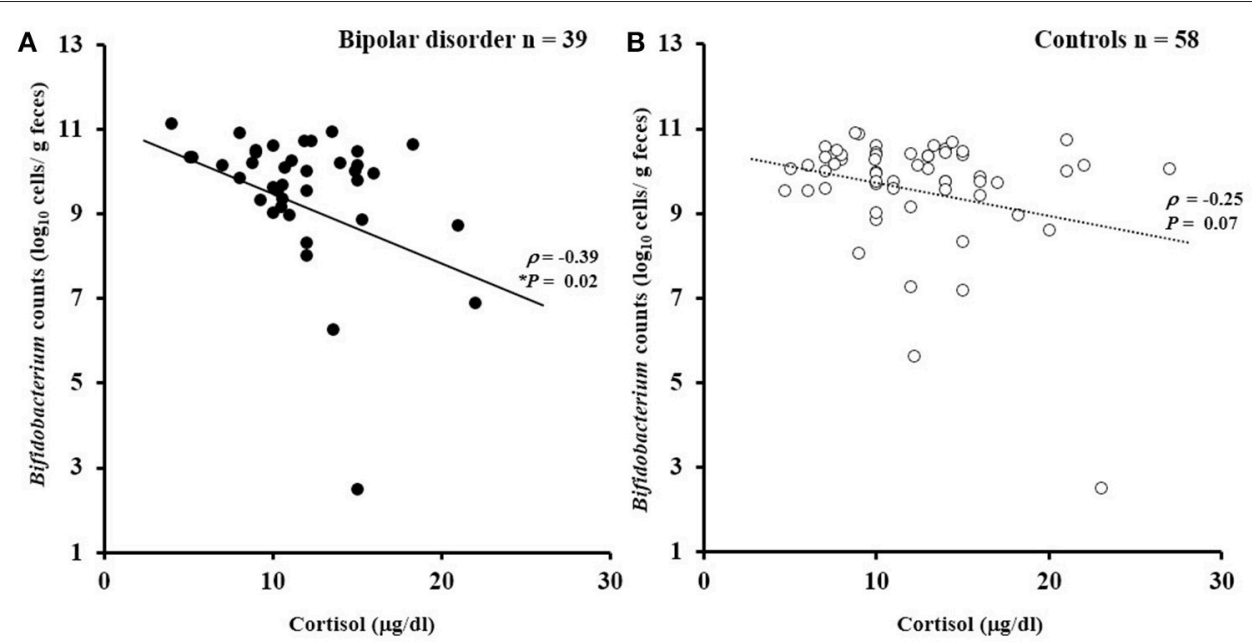

FIGURE 3 | Correlations between Bifidobacterium counts and serum cortisol levels. (A) Bifidobacterium counts exhibited a significantly negative correlation with cortisol levels in the patients with bipolar disorder $(\rho=-0.39, P=0.02)$. (B) A similar trend was observed for the controls $(\rho=-0.25, P=0.07)$.

research (38). A similar non-significant trend $(p=0.07)$ was observed in the controls. Cortisol is involved in the interaction between the brain and various physiological systems, and elevated levels of cortisol have been observed in bipolar disorder (39). As mentioned above, Messaoudi et al. (19) reported that consumption of Bifidobacterium longum R0175 and Lactobacillus helveticus R0052 for 30 days ameliorated distress symptoms and decreased urinary free cortisol levels in healthy volunteers (19). Allen et al. (40) reported that consumption of Bifidobacterium longum 1714 for 4 weeks attenuated stress response to a stressful task in healthy volunteers as reflected by attenuated subjective anxiety and lowered salivary cortisol output $(40,41)$. Our finding of the negative correlation between Bifidobacterium counts and serum cortisol levels is in line with the possible beneficial role of Bifidobacterium on stress response. Although the mechanisms underlying the effect of probiotics on cortisol levels remain unclear, animal studies have shown that the administration of probiotics reduced gut permeability, endotoxemia, neuroinflammation, and hypothalamic expression of corticotropin releasing hormone (CRH), which were accompanied by reduced glucocorticoid levels $(7,42)$. Another study by Takada et al. showed in rats that pretreatment with Lactobacillus casei strain, Shirota, suppressed stress-induced increases in plasma corticosterone, and reduced the number of CRH-expressing cells in the periventricular nucleus of the hypothalamus (43). Further, these changes were possibly related with an increase in gastric vagal afferent activity, suggesting the possible involvement of vagus nerve signaling.

\section{Limitations}

Our study has several limitations. First, as mentioned above, the severity of bipolar disorder in the subjects was relatively mild and further studies are necessary to examine whether severe bipolar cases are related to bacterial counts. Second, we compared only the total counts of Bifidobacterium and Lactobacillus. Both Bifidobacterium and Lactobacillus are comprised of many species, and health-promoting effects may differ depending on the specific species or strains (44). To elucidate the pathophysiological roles of gut microbiota, further studies on other bacteria counts are warranted. Third, the cross-sectional design of the present study makes it difficult to determine whether the observed relationships were causes or effects of the illness. Finally, although we examined the possible correlation between bacterial counts and current medication doses, we did not consider duration of pharmacological treatment in the analyses.

\section{CONCLUSION}

We found no significant difference in Bifidobacterium or Lactobacillus counts between patients with bipolar disorder and controls, suggesting that these bacteria may not play a major role in the pathophysiology of bipolar disorder in our sample. However, the observed negative correlation between Lactobacillus counts and sleep and that between Bifidobacterium counts and serum cortisol levels point to the possible roles of these bacteria in sleep and stress response in the patients.

\section{AUTHOR CONTRIBUTIONS}

EA, HT, TA, TaT, and HK conceived and designed the experiments, analyzed the data, and contributed to the writing of the manuscript. ToT, MO, SY, KH, and NK recruited the subjects, assessed psychiatric symptoms, and gave critical comments to the manuscript.

\section{FUNDING}

This study was conducted using consigned research funds provided by Yakult Central Institute (http://institute.yakult.co.jp/ index_en.php) (HK, Grant number: Jukyo 47). Yakult Central 
Institute provided support in the form of salaries for authors [HT, TA, and TaT], but did not have any additional role in the study design, data collection and analysis, decision to publish, or preparation of the manuscript. The specific roles of these authors are articulated in the author contributions section.

\section{ACKNOWLEDGMENTS}

The authors thank all volunteers for their participation in the study. We also thank Yukiko Kado at the Yakult Central

\section{REFERENCES}

1. Akkasheh G, Kashani-Poor Z, Tajabadi-Ebrahimi M, Jafari P, Akbari $\mathrm{H}$, Taghizadeh $\mathrm{M}$, et al. Clinical and metabolic response to probiotic administration in patients with major depressive disorder:a randomized, double-blind, placebo-controlled trial. Nutrition (2016) 32:315-20. doi: 10.1016/j.nut.2015.09.003

2. Cryan JF, Dinan TG. Mind-altering microorganisms:the impact of the gut microbiota on brain and behaviour. Nat Rev Neurosci. (2012) 13:701-12. doi: $10.1038 / \mathrm{nrn} 3346$

3. Foster JA, McVey Neufeld KA. Gut-brain axis:how the microbiome influences anxiety and depression. Trends Neurosci. (2013) 36:305-12. doi: 10.1016/j.tins.2013.01.005

4. Mayer EA, Tillisch K, Gupta A. Gut/brain axis and the microbiota. J Clin Invest. (2015) 125:926-38. doi: 10.1172/jci76304

5. Gareau MG, Wine E, Rodrigues DM, Cho JH, Whary MT, Philpott DJ, et al. Bacterial infection causes stress-induced memory dysfunction in mice. Gut (2011) 60:307-17. doi: 10.1136/gut.2009.202515

6. Huo R, Zeng B, Zeng L, Cheng K, Li B, Luo Y, et al. Microbiota modulate anxiety-like behavior and endocrine abnormalities in hypothalamicpituitary-adrenal axis. Front Cell Infect Microbiol. (2017) 7:489. doi: $10.3389 /$ fcimb.2017.00489

7. Ait-Belgnaoui A, Colom A, Braniste V, Ramalho L, Marrot A, Cartier C, et al. Probiotic gut effect prevents the chronic psychological stress-induced brain activity abnormality in mice. Neurogastroenterol Motil. (2014) 26:510-20. doi: $10.1111 / \mathrm{nmo} .12295$

8. Macpherson AJ, Harris NL. Interactions between commensal intestinal bacteria and the immune system. Nat Rev Immunol. (2004) 4:478-85. doi: $10.1038 /$ nri1373

9. Mengheri E. Health, probiotics, and inflammation. J Clin Gastroenterol. (2008) 42(Suppl. 3 Pt 2):S177-8. doi: 10.1097/MCG.0b013e31817eedc4

10. Neufeld KM, Kang N, Bienenstock J, Foster JA. Reduced anxiety-like behavior and central neurochemical change in germ-free mice. Neurogastroenterol Motil. (2011) 23:255-64. doi: 10.1111/j.1365-2982.2010.01620.x

11. Sudo N, Chida Y, Aiba Y, Sonoda J, Oyama N, Yu XN, et al. Postnatal microbial colonization programs the hypothalamic-pituitaryadrenal system for stress response in mice. J Physiol. (2004) 558(Pt 1):263-75. doi: 10.1113/jphysiol.2004.063388

12. Perlis RH, Ostacher MJ, Patel JK, Marangell LB, Zhang H, Wisniewski SR, et al. Predictors of recurrence in bipolar disorder:primary outcomes from the systematic treatment enhancement program for bipolar disorder (STEP-BD). Am J Psychiatry (2006) 163:217-24. doi: 10.1176/appi.ajp.163.2.217

13. Geddes JR, Miklowitz DJ. Treatment of bipolar disorder. Lancet (2013) 381:1672-82. doi: 10.1016/s0140-6736:60857-0

14. Fillman SG, Sinclair D, Fung SJ, Webster MJ, Shannon Weickert C. Markers of inflammation and stress distinguish subsets of individuals with schizophrenia and bipolar disorder. Transl Psychiatry (2014) 4:e365. doi: 10.1038/tp.2014.8

15. Maletic V, Raison C. Integrated neurobiology of bipolar disorder. Front Psychiatry (2014) 5:98. doi: 10.3389/fpsyt.2014.00098

16. Muneer A. Bipolar disorder:role of inflammation and the development of disease biomarkers. Psychiatry Investig. (2016) 13:18-33. doi: $10.4306 /$ pi.2016.13.1.18
Institute for her assistance in the analysis of fecal samples and Junko Matsuo, Amiri Noguchi, Anna Nagashima, Ikki Ishida, and Moeko Hiraishi for their assistance in the recruitment of participants.

\section{SUPPLEMENTARY MATERIAL}

The Supplementary Material for this article can be found online at: https://www.frontiersin.org/articles/10.3389/fpsyt. 2018.00730/full\#supplementary-material
17. Watson S, Gallagher P, Ritchie JC, Ferrier IN, Young AH. Hypothalamicpituitary-adrenal axis function in patients with bipolar disorder. $\mathrm{Br} J$ Psychiatry (2004) 184:496-502. doi: 10.1192/bjp.184.6.496

18. O'Mahony SM, Clarke G, Borre YE, Dinan TG, Cryan JF. Serotonin, tryptophan metabolism and the brain-gut-microbiome axis. Behav Brain Res. (2015) 277:32-48. doi: 10.1016/j.bbr.2014.07.027

19. Messaoudi M, Violle N, Bisson JF, Desor D, Javelot H, Rougeot C. Beneficial psychological effects of a probiotic formulation (Lactobacillus helveticus R0052 and Bifidobacterium longum R0175) in healthy human volunteers. Gut Microbes (2011) 2:256-61. doi: 10.4161/gmic.2.4.16108

20. Desbonnet L, Garrett L, Clarke G, Kiely B, Cryan JF, Dinan TG. Effects of the probiotic Bifidobacterium infantis in the maternal separation model of depression. Neuroscience (2010) 170:1179-88. doi: 10.1016/j.neuroscience.2010.08.005

21. Aizawa E, Tsuji H, Asahara T, Takahashi T, Teraishi T, Yoshida S, et al. Possible association of Bifidobacterium and Lactobacillus in the gut microbiota of patients with major depressive disorder. J Affect Disord. (2016) 202:254-7. doi: 10.1016/j.jad.2016.05.038

22. Nguyen TT, Kosciolek T, Eyler LT, Knight R, Jeste DV. Overview and systematic review of studies of microbiome in schizophrenia and bipolar disorder. J Psychiatr Res. (2018) 99:50-61. doi: 10.1016/j.jpsychires.2018.01.013

23. Evans SJ, Bassis CM, Hein R, Assari S, Flowers SA, Kelly MB, et al. The gut microbiome composition associates with bipolar disorder and illness severity. J Psychiatr Res. (2017) 87:23-9. doi: 10.1016/j.jpsychires.2016. 12.007

24. Flowers SA, Evans SJ, Ward KM, McInnis MG, Ellingrod VL. Interaction between atypical antipsychotics and the gut microbiome in a bipolar disease cohort. Pharmacotherapy (2017) 37:261-7. doi: 10.1002/phar.1890

25. Painold A, Morkl S, Kashofer K, Halwachs B, Dalkner N, Bengesser S, et al. A step ahead: exploring the gut microbiota in inpatients with bipolar disorder during a depressive episode. Bipolar Disord. (2018) 26:1-10. doi: $10.1111 /$ bdi. 12682

26. Messaoudi M, Lalonde R, Violle $\mathrm{N}$, Javelot $H$, Desor $D$, Nejdi A, et al. Assessment of psychotropic-like properties of a probiotic formulation (Lactobacillus helveticus R0052 and Bifidobacterium longum R0175) in rats and human subjects. Br J Nutr. (2011) 105:755-64. doi: 10.1017/s0007114510004319

27. Otsubo T, Tanaka K, Koda R, Shinoda J, Sano N, Tanaka S, et al. Reliability and validity of Japanese version of the mini-international neuropsychiatric interview. Psychiatry Clin Neurosci. (2005) 59:517-26. doi: $10.1111 /$ j.1440-1819.2005.01408.x

28. Sheehan DV, Lecrubier Y, Sheehan KH, Amorim P, Janavs J, Weiller $\mathrm{E}$, et al. The mini-international neuropsychiatric interview (M.I.N.I.):the development and validation of a structured diagnostic psychiatric interview for DSM-IV and ICD-10. J Clin Psychiatry (1998) 59 22-33.

29. American Psychiatric Association. Diagnostic and Statistical Manual of Mental Disorders. 4th ed. Washington, DC:American Psychiatric Association (1994).

30. Hamilton M. A rating scale of depression. J Neurol Neurosurg Psychiatry (1960) 23:56-62.

31. Young RC, Biggs JT, Ziegler VE, Meyer DA. A rating scale for mania:reliability, validity and sensitivity. Br J Psychiatry (1978) 133:429-35. 
32. Inagaki A, Inada T, Fujii Y, Yagi G. Equivalent dose of psychotropics. Tokyo:Seiwa Shoten (2013).

33. Matsuda K, Tsuji H, Asahara T, Kado Y, Nomoto K. Sensitive quantitative detection of commensal bacteria by rRNA-targeted reverse transcriptionPCR. Appl Environ Microbiol. (2007) 73:32-9. doi: 10.1128/aem.012 24-06

34. Matsuda K, Tsuji H, Asahara T, Matsumoto K, Takada T, Nomoto K. Establishment of an analytical system for the human fecal microbiota, based on reverse transcription-quantitative PCR targeting of multicopy rRNA molecules. Appl Environ Microbiol. (2009) 75:1961-9. doi: 10.1128/aem.01843-08

35. Serretti A, Mandelli L, Lorenzi C, Pirovano A, Olgiati P, Colombo $\mathrm{C}$, et al. Serotonin transporter gene influences the time course of improvement of "core" depressive and somatic anxiety symptoms during treatment with ssris for recurrent mood disorders. Psychiatry Res. (2007) 149:185-93. doi: 10.1016/j.psychres.2006. 03.020

36. Takada M, Nishida K, Gondo Y, Kikuchi-Hayakawa H, Ishikawa H, Suda K, et al. Beneficial effects of Lactobacillus casei strain shirota on academic stress-induced sleep disturbance in healthy adults:a doubleblind, randomised, placebo-controlled trial. Benef Microbes (2017) 8:153-62. doi: 10.3920/bm2016.0150

37. Miyazaki K, Itoh N, Yamamoto S, Higo-Yamamoto S, Nakakita Y, Kaneda $\mathrm{H}$, et al. Dietary heat-killed lactobacillus brevis sbc8803 promotes voluntary wheel-running and affects sleep rhythms in mice. Life Sci. (2014) 111:47-52. doi: 10.1016/j.lfs.2014.07.009

38. Hellhammer DH, Wust S, Kudielka BM. Salivary cortisol as a biomarker in stress research. Psychoneuroendocrinology (2009) 34:163-71. doi: 10.1016/j.psyneuen.2008.10.026

39. Belvederi Murri M, Prestia D, Mondelli V, Pariante C, Patti S, Olivieri B, et al. The HPA axis in bipolar disorder:systematic review and meta-analysis. Psychoneuroendocrinology (2016) 63:327-42. doi: 10.1016/j.psyneuen.2015.10.014

40. Allen AP, Hutch W, Borre YE, Kennedy PJ, Temko A, Boylan G, et al. Bifidobacterium longum 1714 as a translational psychobiotic:modulation of stress, electrophysiology and neurocognition in healthy volunteers. Transl Psychiatry (2016) 6:e939. doi: 10.1038/tp.2016.191

41. Urita Y, Goto M, Watanabe T, Matsuzaki M, Gomi A, Kano M, et al. Continuous consumption of fermented milk containing Bifidobacterium bifidum YIT 10347 improves gastrointestinal and psychological symptoms in patients with functional gastrointestinal disorders. Biosci Microbiota Food Health (2015) 34:37-44. doi: 10.12938/bmfh.2014-017

42. Ait-Belgnaoui A, Durand H, Cartier C, Chaumaz G, Eutamene H, Ferrier L, et al. Prevention of gut leakiness by a probiotic treatment leads to attenuated HPA response to an acute psychological stress in rats. Psychoneuroendocrinology (2012) 37:1885-95. doi: 10.1016/j.psyneuen.2012.03.024

43. Takada M, Nishida K, Kataoka-Kato A, Gondo Y, Ishikawa H, Suda K, et al. Probiotic Lactobacillus casei strain shirota relieves stress-associated symptoms by modulating the gut-brain interaction in human and animal models. Neurogastroenterol Motil. (2016) 28:1027-36. doi: 10.1111/nmo.12804

44. Stsepetova J, Sepp E, Kolk H, Loivukene K, Songisepp E, Mikelsaar M. Diversity and metabolic impact of intestinal lactobacillus species in healthy adults and the elderly. Br J Nutr. (2011) 105:1235-44. doi: $10.1017 / \mathrm{s} 0007114510004770$

Conflict of Interest Statement: HT, TA, and TaT are employees of Yakult Honsha Co., Ltd.

The remaining authors declare that the research was conducted in the absence of any commercial or financial relationships that could be construed as a potential conflict of interest.

Copyright (c) 2019 Aizawa, Tsuji, Asahara, Takahashi, Teraishi, Yoshida, Koga, Hattori, Ota and Kunugi. This is an open-access article distributed under the terms of the Creative Commons Attribution License (CC BY). The use, distribution or reproduction in other forums is permitted, provided the original author(s) and the copyright owner(s) are credited and that the original publication in this journal is cited, in accordance with accepted academic practice. No use, distribution or reproduction is permitted which does not comply with these terms. 